\title{
Performance of normal hearing school age children on Hindi minimal pair test
}

\begin{abstract}
Speech perception is the process of transforming a continuously changing acoustic signal into discrete linguistic units and is a developmental process having several aspects i.e. Pattern perception, Perception of phonemic contrasts, Vowel \& Sentence perception. There is no standardized minimal pair test material available for testing Hindi school aged children and no normative data for this test is available in Indian scenario. Such normative data will act as a reference for using of Hindi Minimal Pair Test (HMPT, Verma, 2013) test with children with hearing impairment in clinical situation. Hence, this study is warranted. The aim of study is to obtain normative data for school aged children on HMPT of speech perception. A total of 200 participants across two age groups i.e. 6-8 and 8-10 years were included in the study. Each subject underwent Otoscopic examination, Oto Acoustic Emission, Pure tone audiometry, Immittance audiometry and Screening for CAPD. This study indicates perception of minimal pair contrast significantly improves as the age increases. $\mathrm{P}$ value obtained for comparison between males and females is 0.62 which is greater than 0.05 suggesting no significant difference between mean scores of male and female participants. For age comparison obtained $p$ value is 0.00 , suggesting a significant difference between the two age groups. $\mathrm{P}$ value of 0.051 for interaction suggests no significant interaction between age and sex statistically.
\end{abstract}

Keywords: Speech Perception, Auditory discrimination, Immittance audiometry
Volume I2 Issue I - 2020

\author{
Subhasmita Sahoo,' Aparna Nandurkar² \\ Department of Audio-Vestibular Medicine, Institute of Health \\ Sciences, India \\ Department of Audiology, AYJNISHD (D), India
}

Correspondence: Subhasmita Sahoo, Department of AudioVestibular Medicine, Institute of Health Sciences, Bhubaneswar, Odisha, India, Email subhu2I sahoo@gmail.com

Received: December 19, 2019 | Published: January 17, 2020
Abbreviations: HMPT-Hindi minimal pair test; SCAPScreening for Central Auditory Processing Disorder; OAE-Oto Acoustic Emission

\section{Introduction}

Speech perception is the process of transforming a continuously changing acoustic signal into discrete linguistic units, ${ }^{1}$ and is a developmental process having several aspects i.e. Pattern perception, Vowel perception, Perception of phonemic contrasts, Sentence perception. Due to this ability of speech perception, humans acquire language which makes them unique as they can communicate using structured codes of a language. The ability to develop and use oral language is closely related to the ability to process speech through the sense of hearing. This relationship is well established from language delays seen among children with hearing impairments. In order to lessen the effect of loss and enhance the development of auditory verbal language in children with hearing impairment, clinicians and researchers need to understand the limitations imposed by hearing loss on auditory perception of speech. ${ }^{2}$ The term phoneme (from Ancient Greek phonema, "sound made, utterance, thing spoken, speech, language") was reportedly first used by A. Dufriche-Desgenettes, ${ }^{3}$ but it referred only to a speech sound. Phoneme is the smallest linguistic unit of a language. A phoneme is one of the units of sound that distinguish one word from another in a particular language. Phoneme perception is a form of auditory perception in which the listener and speaker distinguish among the sound contrasts in a language. Auditory discrimination includes the ability to contrast sounds in the environment as well as the sounds and their patterns in language. Auditory discrimination is an ability to discriminate between the sounds at the articulatory, acoustic and other cognitive levels.
For speech perception throughout human life, cues or features of sounds are required to understand and process it, which will result in correct production and perception. A "cue" is a specific aspect of an acoustic signal which is important for recognizing a phonetic segment, or for distinguishing between two phonetic segments as stated by Dorman. ${ }^{4}$ Cues differentiate sounds that are produced at different places of articulation or manners of articulation. The speech system combines these cues to determine the category of a specific speech sound. Consonants are produced with a greater degree of constriction as compared to vowels. Vowels and consonants are also classified using different sets of features.

Raja, Rathna Kumar, Prakash and Reddy ${ }^{5}$ compared the perception of phoneme contrast among 8 to 12 year old children with normal hearing (NH), Cochlear Implants (CI), and Hearing Aids (HA) in Telugu and reported $86 \%$ correct consonant contrast scores for children with NH in auditory-only condition. Assessing the auditory perception skills in children, it is pre-emptory that a clinician should consider speech perception as a principal skill to be tested. Speech perception must be routinely assessed with a battery of tests, so that, a prognosis regarding the development of speech, language, reading, and cognitive skills can be established. Speech perception assessment plays an essential role in comprehensive audiological evaluation. Information from speech perception testing is fundamental to planning (re)habilitation and educational strategies for young children with hearing loss. Assessment of young children substantially differs from that of older children and adults, and hence, these differences must be reflected in the content validity of speech perception assessment tools for Pediatric population. 


\section{Need for the study}

Most of the standard test material available for speech perception testing in children has been developed in the western countries and is available in English. There is a dearth of speech perception material for children in Indian languages, especially Hindi. There is no standardized minimal pair test material available for testing Hindi school aged children and no normative data for this test is available in Indian scenario. Such normative data will act as a reference for using of Hindi Minimal Pair Test (HMPT) test with children with hearing impairment in clinical situation. Hence, this study is warranted.

\section{Aim of the study}

To obtain normative data for school aged children on Hindi Minimal Pair Test (HMPT) of speech perception.

\section{Objectives}

i. To administer Hindi Minimal Pair Test on 6-10 year old school aged children with normal hearing.

ii. To compare the performance on Hindi Minimal Pair Test in terms of age (6 to 8 and 8 to 10 years).

iii. To compare the performance on Hindi Minimal Pair Test in terms of gender in each age group.

\section{Hypotheses}

a) There will be no significant difference in performance of 6-8 and 8-10 year old normal hearing children on the Hindi Minimal Pair Test.

b) There will be no significant difference in performance of male vs female school aged normal hearing children on the Hindi Minimal Pair Test.

\section{Methodology}

\section{Participants}

A total of 200 participants were included from regular schools in Mumbai and its suburbs with Hindi speaking background and were further divided into Group-1 (6-8 years) and Group-2 (8-10 years). 100 participants in each of the two groups ( $6+-8$ years, $8+-10$ years) having 50 males and 50 females each, were included in the study.

\section{Tool}

The tool used for this study was Hindi Minimal Pair Test developed by Verma (2013) which consists of one list with 42 test plates and 2 practice plates. Each plate contains 2 pairs i.e. 4 items, one target pair and one foil pair which is randomly placed on A4 size paper. The test was recorded by a native male adult speaker to be presented at $60 \mathrm{~dB}$ HL. Closed set format were used where the subject has to listen to the stimulus and point to the corresponding picture out of four pictures on the plate. Each correct response will be provided with " 1 " point and " 0 " will be allotted for each wrong answer.

\section{Inclusion criteria}

a) Age range of 6 to 10 years.

b) No associated impairments.

c) Both male and female subjects. d) From Hindi speaking background (Hindi spoken at home and school).

e) Age appropriate speech and language development as ascertained by MECD.

f) Normal otoscopic findings.

g) No history of or complaint of middle ear infection.

h) Bilateral Pass result on OAE screening.

i) Pure tone thresholds of less than or equal to $20 \mathrm{~dB} H L$ in both ears.

j) Pass on SCAP (Yathiraj \& Mascarenhas, 2003).

\section{Procedure}

All participants and their parents were given a brief overview of the test procedure. Written consent on the consent form was mandatory prior to initiating any test procedure. Anyone who did not fulfill the inclusion criteria was not included in the study. Otoscopy was done to check for any external ear abnormalities like excessive wax or foreign bodies; tympanic membrane perforation or ear discharge. Transient Evoked Oto Acoustic Emission (TEOAE) was done to check for any abnormality of the peripheral auditory system up to the level of outer hair cells. Pure tone audiometry was conducted in a sound treated room with ambient noise levels within permissible limits according to ANSI standards (ANSI S3.6-ISO389). Air conduction thresholds were determined using GSI 61 audiometer with TDH 39 earphones. Air conduction thresholds were determined at octave frequencies from $250 \mathrm{~Hz}$ to $8 \mathrm{KHz}$ using the modified Hughson Westlake procedure. Immittance audiometry was done to ensure normal middle ear functioning using GSI 38 immittance audiometer. Tympanometry was done to determine the static acoustic admittance and ear canal volume. Reflexometry was done at $500 \mathrm{~Hz}, 1000 \mathrm{~Hz}, 2000 \mathrm{~Hz}$ and $4000 \mathrm{~Hz}$ to ensure the presence of acoustic reflexes. A standard 226 $\mathrm{Hz}$ probe tone stimulus was used for both tympanometry and acoustic reflex testing.

\section{Administration of the Hindi Minimal Pair Test (HMPT)}

The subjects were instructed that words will be presented from the loudspeaker, at the same time the pictures will be shown in front of you. They will have to listen carefully to the targeted word and point to the respective picture. For the ease of understanding, two practice plates were administered in similar condition and these were not be scored. This helped to judge whether the subject had understood the task. The test was administered in a quiet one room setup, directly through laptop connected to speakers. In order to calibrate the test setup, the recorded stimuli were played via a DELL laptop connected to Xpro 2.0 loudspeakers placed at $45^{\circ}$ azimuth. The microphone of a Bruel \& Kjaer- Type2250 Sound Level Meter (SLM) was held at a distance of $1 \mathrm{~m}$ from the speakers and measurements were made as the stimuli were played from the laptop. The SLM was set on "fast" mode. The volume of the speaker was manipulated till readings between 55-60 dB SPL were consistently obtained on the SLM. The volume setting on the speaker which resulted in this intensity was marked to enable consistent setting during test presentation. Closed set format was used where the subject has to listen to the stimulus and point to the corresponding picture out of four pictures on the plate as per the instructions given. For each test plate, two stimulus items were presented presented one after another. The recorded stimulus 
was paused after every word and the child was given time to respond to the stimulus. In case the child did not respond the stimulus was played once more. The HMPT consists of 42 target minimal pairs, one pair on each test plate. Maximum obtainable score was 84 . The total time taken for the entire procedure was approximately 30 minutes per participant.

\section{Statistical analysis}

Since the sample consisted of a large number of children $(200$ participants), assuming normal distribution of scores, parametric tests were applied. Descriptive statistics were obtained by calculating the means of scores obtained by the subjects in the four sub groups across the two groups. Two-way ANOVA was performed to study the effect of age and gender. This was followed by post-hoc analysis for multiple comparisons of the four age groups using Bonferroni corrections. Independent samples t test was performed to compare the scores of males and females.

\section{Results}

HMPT has 42 target minimal pairs, hence the maximum obtainable score is 84 . The mean score of $6+$ to 8 year old males was 75.48 with a standard deviation of 4.02 and the mean score of females was 76.72 with a standard deviation of 4.20 while the mean score for $8+$ to 10 year old males was 80.38 with a standard deviation of 2.60 and the mean score for females was 79.64 with a standard deviation of 3.22. SD values for female participants are higher compared to male participants. Also the SD values are higher for age group 6+-8 years than $8+-10$ years age group. Female participants performed better than male participants for $6+-8$ years age group while male performed better for $8+-10$ years age group. Tables $1 \& 2$ depicts minimum scores obtained for male participants are higher than those for female participants in all groups while the maximum scores obtained for female participants are higher than those of male participants in all groups. From the Graph 1 it is also evident that scores of $6+-8$ year age group were lower compared to the $8+-10$ years age groups.

Table I Details of participants included in the study

\begin{tabular}{lllll}
\hline Age Groups & Groups Mean age (years) & SD & Number & \\
\hline $6+-8$ years & 6.95 & 0.62 & Male $=50$ & Female $=50$ \\
$8+-10$ years & 9.01 & 0.63 & Male $=50$ & Female $=50$ \\
\hline
\end{tabular}

Table 2 Comparison of the scores of two age groups of males and females

\begin{tabular}{lllllllll}
\hline & Mean & & SD & & \multicolumn{2}{c}{ Minimum score } & Maximum score \\
\cline { 2 - 9 } Age groups & Male & Female & Male & Female & Male & Female & Male & Female \\
& 75.48 & 76.72 & 4.02 & 4.20 & 63 & 62 & 82 & 83 \\
$6+-8$ years & 80.38 & 79.64 & 2.60 & 3.22 & 73 & 71 & 84 & 84 \\
$8+-10$ years & & & & & & & & 83 \\
\hline
\end{tabular}

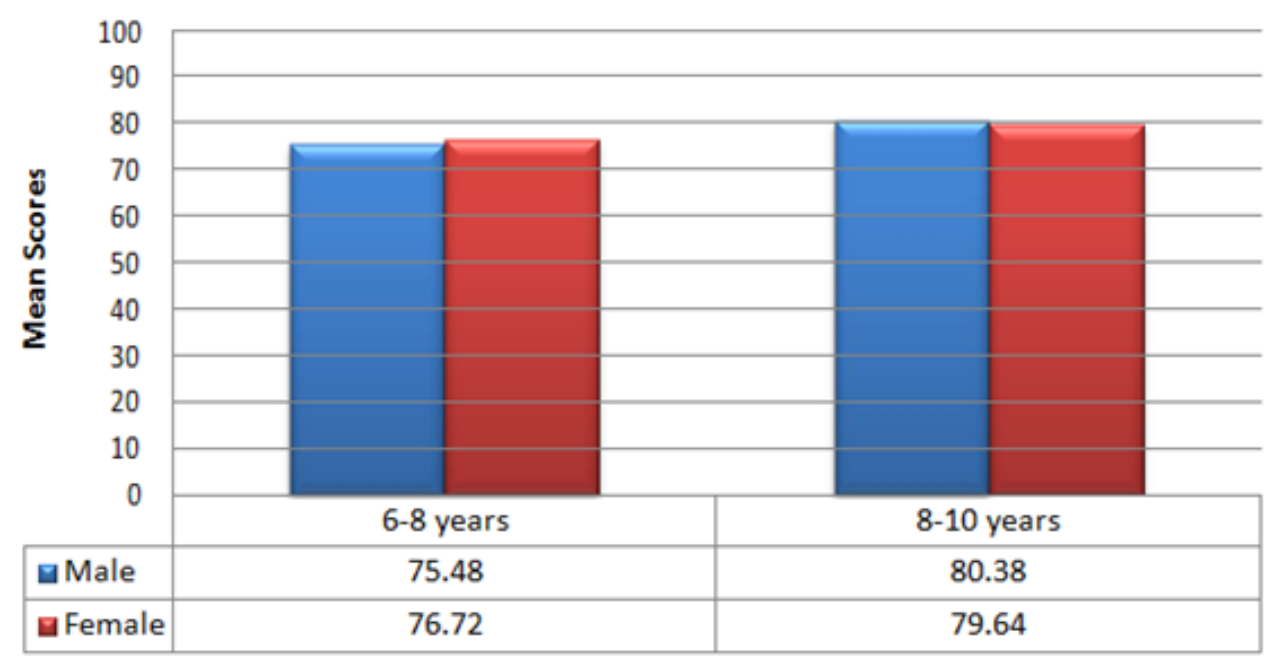

Age Groups

Graph I Mean Scores obtained by different age groups of males and females. 
In order to determine the effect of age, gender as well as the combined effect of age and gender on the mean scores a two way ANOVA test was performed. P value obtained for comparison between males and females is 0.62 which is greater than 0.05 suggesting no significant difference between mean scores of male and female participants. For age comparison obtained $p$ value is 0.00 , suggesting a significant difference between the two age groups. $\mathrm{P}$ value of 0.051 for interaction suggests no significant interaction between age and sex statistically. But as we can see from graph-1 there is an interaction between age and sex. In the age group 6 to 8 years females outperformed the age matched males, however males outperformed the age matched female for 8 to 10 years age group. Table 3 depicts the results of the two-way ANOVA, based on this the hypothesis 1) That, "There will be no significant difference in performance of 6-8 and 8-10 year old children on the Hindi Minimal Pair Test is rejected. Further, hypothesis 2) that "There will be no significant difference in performance of male vs female school aged children in each group on the Hindi Minimal Pair Test is accepted.

It may also be noted that none of the groups obtained ceiling scores. On perusal of scores of individual participants too it was observed that none of the participants obtained $100 \%$ scores. This could imply that the perception of minimal pair contrasts is not acquired in Hindi speaking children by 10 years of age. The obtained data was further analyzed to obtain the percent correct responses for the various contrasts tested by the minimal pairs on the HMPT. In 6-8 years, Affricate-Fricative and Lateral-Stops obtained the scores between $95-100 \%$, which suggest that these contrasts were comparatively easier than other distinctive features of minimal pair. Stop-Trill, Fricative-Nasal, Trill-Fricative, Trill-Semivowel, Stop-Fricative, Stop-Nasal and Stop-Affricates achieved 90- 95\% score. Affricate-Nasal and Aspiration achieved less than $90 \%$ score which suggest higher level of difficulty for these minimal pair contrast. In 8-10 years, Affricate-Fricative, LateralStop, Stop-Trill, Fricative-Nasal, Trill- Fricative, Trill-Semi vowel, Stop-Fricative and Stop- Nasal achieved $95-100 \%$, which suggest that these contrasts were comparatively easier than other distinctive features of minimal pair. Stop-Affricate and Affricate-Nasal obtained the scores between $90-95 \%$. The score on Aspiration is less than $90 \%$ which suggest higher level of difficulty. Score of Voicing is $90 \%$ for the age group of 6-8 years and $94.2 \%$ for $8-10$ years. Place obtained $89.9 \%$ for the age group of $6-8$ years and $95 \%$ for 810 years. Hence for both the age groups i.e. 6-8 and 8-10 years, Aspiration is more difficult contrast than other features of minimal pair.

Table 4 shows the percentage of correct responses obtained for the various contrasts tested along with the number of stimuli for each of the contrasts for the two age groups. From the Table 4, it is evident that perception of minimal pair contrast significantly improves as the age increases. Most manner contrasts obtained higher scores than those for voicing and place for both the age groups i.e. 6-8 and 8-10 years.

Table 3 Results of two-way ANOVA

\begin{tabular}{lllllll}
\hline $\begin{array}{l}\text { Source of } \\
\text { Variation }\end{array}$ & SS & DF & MS & F & P-value & F crit \\
\hline Gender & 3.12 & I & 3.12 & 0.24 & 0.621 & 3.88 \\
Age & 764.40 & I & 764.40 & 59.88 & 0.000 & 3.88 \\
Interaction & 49.00 & I & 49.00 & 3.83 & 0.051 & 3.88 \\
Within & 2501.86 & 196 & 12.76 & & &
\end{tabular}

Table 4 Analysis of correct response for different minimal pair contrast

\begin{tabular}{|c|c|c|c|}
\hline Features tested & $\begin{array}{l}\text { No. of stimulus } \\
\text { items }\end{array}$ & $6-8$ years & 8 - 10 years \\
\hline \multicolumn{4}{|l|}{ Manner } \\
\hline Affricate - Fricative & I & $96 \%$ & $97.5 \%$ \\
\hline Lateral - Stop & I & $95.5 \%$ & $98.5 \%$ \\
\hline Stop-Trill & 5 & $94.7 \%$ & $97.7 \%$ \\
\hline Fricative - Nasal & 4 & $94.7 \%$ & $97.6 \%$ \\
\hline Trill - Fricative & 3 & $93.1 \%$ & $97.1 \%$ \\
\hline Trill- Semi vowel & 1 & $93 \%$ & $97 \%$ \\
\hline Stop - Fricative & 4 & $91.7 \%$ & $96.2 \%$ \\
\hline Stop - Nasal & 5 & $90.6 \%$ & $96.5 \%$ \\
\hline
\end{tabular}

\begin{tabular}{lcll}
\hline Stop - Affricate & 4 & $90.3 \%$ & $93.5 \%$ \\
Affricate - Nasal & 4 & $84.5 \%$ & $91.6 \%$ \\
Aspiration & 2 & $74.2 \%$ & $85.2 \%$ \\
Voicing & 2 & $90 \%$ & $94.2 \%$ \\
Place & 6 & $89.9 \%$ & $95 \%$ \\
\hline
\end{tabular}

\section{Discussion}

\section{Age effects}

The results of statistical analysis showed significant difference between the scores obtained by the two age groups i.e. 6 to 8 year and 8 to 10 year old age groups on administration of HMPT. The results also showed that the highest scores were obtained by 8 to 10 year olds and the lowest scores were achieved by the 6 to 8 year olds. These results clearly indicate that the performance improves with the increase in age. Male participants performed poorer than female participants for the age group of 6-8 years but SD values decreased and mean score 
increased with increase in age, which suggests that males performed better than females for the age group of 8-10 years. Also the difference between performance of male and female participants decreased with increase in age. However, even participants from the highest age group did not obtain ceiling scores, suggesting that certain features of minimal pair contrast are not completely achieved by 10 years of age in Hindi speaking children. Raja, Rathna Kumar, Prakash and Reddy ${ }^{5}$ investigated perception of phoneme contrasts in 15 children with normal hearing within the age range of 8 to 12 years. They compared perception of vowel and consonant contrasts in Telugu language using the auditory-only and auditory-visual modality. They used $\mathrm{ABX}$ paradigm to present minimal pairs via the loudspeakers at 60 $\mathrm{dB}$ SPL in a sound field condition and found that children performed better for vowel contrasts than for consonant contrasts. They reported mean percentage scores on $85 \%$ and $86 \%$ for the vowel and consonant contrasts respectively for the auditory-only condition. For auditoryvisual condition mean percentage scores were $92 \%$ for both the vowel and consonant contrasts. These results indicate that both vowel and consonant contrasts in minimal pairs were not developed in these participants till the age of 12 years.

\section{Effect of gender}

The results of statistical analysis showed no significant difference in the performance of the two gender groups i.e. Males and Females. It was observed that female participants performed better compared to male participants for the age group of 6-8 years while males performed better compared to female participants for the age group of 8-10 years. Robinson ${ }^{6}$ studied the effect of talker age and gender on speech perception of pediatric hearing aid users; they found that for a closedset vowel identification task, the talker-type did not significantly affect percent-correct scores. However, this effect was seen for a $\mathrm{CNC}$ word recognition task. A significant gender effect was also seen stating that the average percent- correct word score for the adult male was $79 \%$ compared to an average score of $66 \%$ for females. A similar pattern was obtained for the phoneme scores. In males acquisition of speech and language is slightly slower in early years than females but as age increases they perform equal or better than females. Such gender differences could be found because of anatomical changes, women seem to be wired more for socialization and memory while men appear geared toward perception and coordinated action. The female brain appears to have increased connections between neurons in the right and left hemispheres of the brain, and males seem to have increased neural communication within hemispheres from frontal to rear portions of the organ.

\section{Performance on different minimal pair contrast}

In current study, it was observed that minimal pair contrast significantly improves as the age increases. Manner contrasts obtained higher scores than voicing and place. Affricate-Fricative and LateralStops obtained the higher scores than Stop-Trill, Fricative-Nasal, Trill-Fricative, Trill-Semi vowel, Stop-Fricative, Stop-Nasal, StopAffricates, Affricate-Nasal and Aspiration. And also final consonant contrast is more difficult than initial consonant contrast. Voiceless sounds were perceived better than voiced sound, perception of nasals and fricatives were superior to stops whereas percentage of performance for perception of alveolars and bilabials were better than velar in auditory and visual mode. It was also found that the duration feature was perceived better through audiovisual and visual modalities than through auditory modality. ${ }^{7-10}$

\section{Conclusions}

A. As indicated by the significant difference between the means scores, there is a statistically significant difference in the performance of the two age groups indicating that speech perception performance improves with age i.e. 6 to 8 year old and 8 to 10 year old children on HMPT.

B. Though there was a difference between the mean scores, there was no statistically significant difference in the performance of the Male and Female participants on HMPT; however, females performed better than males for 6-8 years while males performed better for 8-10 years.

C. The Hindi Minimal Pair Test can be successfully used in school aged children with normal hearing and it has a potential to be a useful clinical tool as a part of a test battery.

D. Results of feature analysis reveals perception of minimal pair contrasts significantly improves as the age increases. Manner contrasts obtained higher scores than voicing and place in both the age groups i.e. 6-8 and 8-10 years.

\section{Implications of the study}

1. The study utilizes a closed set test of speech perception using minimal pairs for normal hearing school aged children in the age range of 6 to 10 years. The normative data obtained through the study can be used for clinical assessment of speech perception for this age group.

2. The test can be used for obtaining information about acoustic cues and to assess if the children can differentiate different distinctive features.

3. The test can be used to assess phoneme perception skills in children with hearing impairment and can be useful in assessing the measure of delay from the normal hearing peers.

4. Feature analysis of the performance done in the present study can help in tracking the development of perception of minimally contrasting pairs in typically developed children.

\section{Summary}

Speech perception plays an important role in acquisition of spoken language as it helps us to make sense of what we hear and indirectly thus helps us to speak. Thus assessment of speech perception has become a key component of the audiological test battery. There are various tests available for testing speech perception skills in children however there are very few tests which can be used in the Indian context. This necessitates development of test in Indian context, especially in Hindi as it is the most widely spoken language. Hindi Minimal Pair Test can be successfully used to assess speech perception skills in school aged children with normal hearing. This test has a potential to be a useful clinical tool to assess speech perception.

\section{Acknowledgments}

I take this opportunity with much pleasure to thank all the people who have helped me through the course of my journey towards the completion of my research.

The two most wonderful persons on this earth furnished me with all the facilities and joys one can dream for. They are my "Baba and 
Bou...” My parents!!! There are no words in this world to describe my gratitude towards you. Love u „MUMMY-PAPA“!!!

I seize this opportunity to extent my heartfelt thanks to my guide,

Dr. Aparna N Nandurkar, who worked and supported me aroundthe-clock with an all-out effort to get this project submitted. I'ell always wonder what the source of your endless energy and motivation is. Thank you very much ma"am.

My special thanks to Sarita Rautra maeam for constant guidance and for showing me the right path over the years.

I also would like to express my gratitude to my Director, his painstaking efforts and guidance has helped me reach this level.

\section{Conflicts of interest}

There are no conflicts of interest.

\section{Funding}

None.

\section{References}

1. Rvachew S, Grawburg M. Correlates of phonological awareness in Preschoolers with speech sound disorders. J Speech Lang Hear Res. 2006;49(1):7487.

2. Kirk KI, Diefendorf AO, Pisoni DB, et al. Assessing speech perception in children. Audiologic evaluation and management and speech perception assessment, 1997. p. 101-132.
3. Dufriche Desgenettes A. Sur la nature des consonnes nasales. Bulletin de la Society de Linguistique de Paris. 1873;2(8):63.

4. Dorman M. Speech Perception by Adults. In RS Tyler editor. Cochlear Implants. Audiological Foundations. London: Whurr; 1995. p. 145-190.

5. Raja S, Rathna Kumar SB, Prakash SGR, et al. Perception of Phoneme Contrast in Children with Hearing Impairment in Telugu. Language in India. 2010;10:129-145.

6. Robinson EJ. The effect of talker age and gender on speech perception of pediatric hearing aid users. Psychology. 2011.

7. Garima B. Contribution of auditory \& visual modalities in speech perception of hearing impaired children. Unpublished master"s thesis, Osmania University, Hyderabad, India, 1998.

8. Jusczyk PW, Cutler A, Redanz NJ. Infants' preference for the predominant stress patterns of English words. Child development. 1993;64(3):675-687.

9. Robbins AM, Kirk KI. Speech perception assessment and performance in paediatric cochlear implant users. Seminars in hearing. 1996;17:353-369.

10. Verma. Development of speech perception test material using Hindi minimal pairs for children with hearing impaired childrenUnpublished MSC dissertation, AYJNIHH Mumbai. 2013. 